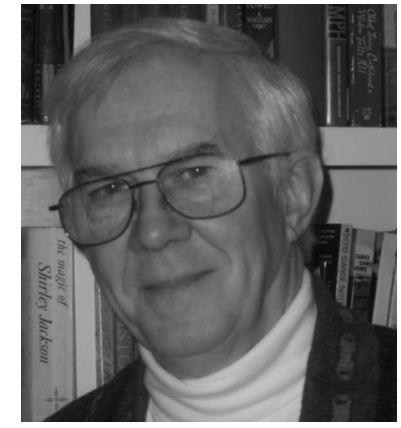

\title{
Metamaterials and Photonic Crystals in Warsaw
}

Sometimes the future reveals itself in unexpected ways. This time it popped up in the lecture halls and classrooms of the Warsaw Institute of Technology this past September. I was there as a member of the organizing committee for the SPIE International Congress on Optics and Optoelectronics. I am a bit embarrassed by how little I had to do, but I was highly impressed by the skill and effort of the Polish chapter of SPIE that went into organizing a first-rate conference during a week that celebrated the formation of the Solidarity movement in Poland. The contrast between the struggle that began 25 years ago in Gdansk and this conference in Warsaw with 700 participants (including about 160 students) representing 44 countries from all the 6 continents was breathtaking.

The first day was devoted to a plenary session titled "Hot Topics in Optics and Optoelectronics." The talks highlighted some of the areas addressed during the Congress. They included liquid crystals, photonic crystals and photonic fibers, metamaterials, integrated optics, and lasers and their applications. What struck me as I listened to the Hot Topic talks and the presentations during the week was how much of the research depended on man-made structures.

As optics expanded beyond the confines of lenses and imaging, researchers first depended on natural crystals for devices such as semiconductor detectors and x-ray crystal spectrometers. Later, changes were made by adding dopants that changed the electronic properties, but they did

not change the physical properties much. It was, I think, when Henry Rowland invented the ruling engine to fabricate periodic structures, diffraction gratings, that could not be found in nature that engineering was introduced into optics. Today, with computer-controlled integrated circuit techniques, structures (almost all being labeled "nanostructural," even at the lower end of the microstructure scale) are produced that show physical effects that are ripe for development into devices. By drawing highly intricate arrangements of glass tubes and cylinders into elaborate photonic fibers, the nature and quality of short pulses can be controlled as never before. The fabrication of these metamaterials and structured fibers should provide incredible opportunities for material and optical engineering.

As I bounced from conference to conference, it appeared to me that the debris from the dotcom bust has rained down upon optics and optical engineering providing us with a whole new set of effects, phenomena, and devices. Right now, based on the conference presentations I heard, I would estimate that, for some time to come, the community will be inventing the most promising structures and exploring their physics by determining the theoretical and fabricational limitations.

Amid these descriptions of advances there was a note of caution expressed by Tomasz Szoplik of Warsaw University. He noted that he and most of his colleagues at the conference could only contribute to the theory and analysis of these novel structures because research funds are limited and that money for facilities was nonexistent. Researchers in these new fields do what they can by collaborating with others. But as any researcher who depends on the kindness of collaborators knows, you miss the additional insights into the structures you are studying if someone else fabricates them for you. Nor can you abort the sequence of planned experiments to pursue a line of investigation that is more promising.

Perhaps now that these countries are part of the European Union, they will have access to research funding beyond what their home countries can provide. Funding of a fabrication facility in these EU countries could establish a new center of innovation by a technically strong and enthusiastic optical engineering community.

Donald C. O'Shea Editor 